Original Article

\title{
AWARENESS, KNOWLEDGE AND ATTITUDE ABOUT DENGUE AMONG SOCIETY
}

\author{
PATIL P. J., PATIL V. S., CHAUDHARI R. D., MALU J. S. \\ Department of Pharmacology, Marathwada Mitra Mandal's College of Pharmacy, Pune 411033 (M. S.) \\ Email: respjpatil@gmail.com
}

Received: 10 Jul 2020, Revised and Accepted: 08 Sep 2020

\begin{abstract}
Objective: The survey about dengue was carried out to gather the information about dengue fever, create awareness among the people. To determined the index of knowledge, attitude and awareness among the surveyed population about dengue.

Methods: The questionnaire was prepared under the guidance of a pharmacologist. Pilot study was conducted among ten nos. of the population prior actual study. Questionnaire was revised and finalized according to an input of the pilot study. This questionnaire presented to the hundred peoples of different age group. Method involves face to face interview and seeking answers for different questions. Responses were recorded for further analysis and interpretation. Responses were sorted and analyzed. Different questions were categorized into the domain of awareness, knowledge and attitude. Data was presented in the form of graphs.
\end{abstract}

Results: Percentage of awareness, knowledge and attitude found to be 59, 44, 52 respectively. It is observed that three parameters are almost in the range of fifty percent. Precautions plays vital role in prevention of Dengue.

Conclusion: it is concluded that there is scope for improvement for creating awareness, knowledge and attitude among the surveyed population as representative of the general population. This may help to overcome the risk of this communicable and deadly disease.

Keywords: Awareness, Dengue among

(C) 2020 The Authors. Published by Innovare Academic Sciences Pvt Ltd. This is an open access article under the CC BY license (http://creativecommons.org/licenses/by/4.0/] DOI: http://dx.doi.org/10.22159/ijcpr.2020v12i6.40278. Journal homepage: https://innovareacademics.in/journals/index.php/ijcpr

\section{INTRODUCTION}

Dengue fever, also called as break-bone fever, is a mosquito-borne infection that causes a severe flu-like illness. There are four types of viruses that can cause dengue fever, all of which spread by a certain type of mosquito. Dengue can vary from mild to severe and severe forms include dengue shock syndrome and dengue hemorrhagic fever (DHF). According to the WHO 2.5 billion people, or two-fifths of the world's population, are now at risk from dengue. The disease is now endemic in over 100 numbers of countries. Dengue hemorrhagic fever is leading cause of serious illness and death among children in the number of Asian countries. In 2007, there were over 890,000 numbers of reported cases of dengue in the Americas, of which 26,000 cases were DHF. Dengue infection rates among the people who have not been previously exposed to the virus are commonly $40 \%$ to $50 \%$ during epidemics but may sometimes reach $80 \%$ to $90 \%$. Now a day's approximately half-a-million people with DHF are hospitalized each year, of which many are children. About $2.5 \%$ of these patients die. DHF fatality reads may exceed $20 \%$ if untreated. If there is access to medical care with health care professionals are trained in treating DHF, the death rate may be less than $1 \%$. A symptom is something the patient feels or/and reports, while a sign is something that other people, including the doctor, detects. A headache an example of a symptom, while a rash may be an example of a sign [1-3]. Causes of Dengue-There are four Dengue viruses that cause Dengue fever, all of which are spread by a species of mosquito known as the Aedes aegypti mosquito, and rarely by the Aedes albopictus mosquito. Aedes aegypti was originated in an African country, but nowadays is found in all tropical areas around the world and prospers in and close to areas of the human population. High-risk regions for dengue fever are South America, Central America, the Caribbean and tropical Asia; specifically-northern Argentina, northern Australia, the entirety of Bangladesh, Barbados, Bolivia, Brazil, Cambodia, Dominican Republic, Costa Rica, Guatemala, Guyana, Honduras, India, Indonesia, Jamaica, Laos, Mexico, Malaysia, Micronesia, Pakistan, Panama, Paraguay, Puerto Rico, Philippines, Samoa, Singapore, Sri Lanka, Taiwan, Suriname, Thailand, Trinidad, Venezuela and Vietnam, and increasingly in southern China [4-6]. Diagnosis of Dengue fever:Assess the symptoms-the doctor will take into account all your symptoms to properly diagnose whether you have dengue. Some tests ordered to determine whether it is dengue infection or other. Blood sample-this sample can be tested in a laboratory in a number of ways to find signs of the dengue virus. If virus is found diagnosis is straightforward; if this fails there are other blood tests which identify antibodies, antigens and nucleic acids and including ELISA (enzymelinked immunosorbent assay), HI assay (hemagglutination inhibition assay) and RT-PCR (reverse transcriptase-polymerase chain reaction), Assess your medical history-The doctor will need to know travel history and medical history of the patient, if it involves mosquito exposure [7-9]. Milder forms of dengue treatment methods are: Prevent dehydration-high fever and vomiting it can dehydrate the body of patient. Make sure drink clean water rather than tap water. Rehydration salts also help replace fluids and minerals. Painkillers-this can help lower fever and pain. As some non-steroidal antiinflammatory drugs, such as aspirin or ibuprofen, can increase the risk of internal bleeding, patients are advised to use Paracetamol instead. For More severe forms of dengue fever: Intravenous fluid supplementation (IV drip)-in some harsher cases of dengue the patient is unable to take fluids via the mouth) and will need to receive an IV drip. Blood transfusion-a blood transfusion recommended for patients with severe dehydration. Hospital care-it is important that patients treated by medical professionals, this way you can be properly monitored (e. g. blood pressure, fluid levels) in case your symptoms worsen. If the patient is cared for by physicians and nurses experienced with the effects and complications of hemorrhagic fever, lives can be saved [10]. The best method of prevention is to avoid being bitten by mosquitoes. Clothing-your chances of being bitten are reduced if you expose as little skin as possible. Mosquito repellants-be sure to use one with at least $10 \%$ concentration of DEET, avoid using DEET on young children. Use mosquito traps or nets-studies have shown that the risk of being bitten by mosquitoes is considerably reduced if you use a mosquito net when you go to sleep. Smell-Avoid wearing scented soaps and perfumes. Windows-use structural barriers, such as window screens or netting. Camping-if you are camping, treat clothes, shoes and camping gear with permethrin [11]. Dengue viruses are transmitted by the bite of an infected Aedes (subgenus Stegomyia) mosquito Virus. It can be identified by the scale patterns or white bands on its legs and thorax Hun; the A aegypti 
mosquito is the most common epidemic vector for spread of dengue man's serve as the primary reservoir for dengue. India contributes for $34 \%$ of the global burden of dengue. Success of dengue control depends largely on correct attitude towards the practice of preventive measure [12]. Dengue prevention and control solely depends on effective vector control measure. The best preventive measure is to eliminate the place where the mosquito lays eggs. Container holding water for long are among common breeding sites [13].

\section{MATERIALS AND METHODS}

Literature survey was carried out to gather information about dengue fever and create awareness about dengue among society. Questionnaire containing different eleven questions were prepared by pharmacologist. Questionnaire is presented in the table 2.
Questionnaire contains different aspects of study viz. awareness, knowledge, and attitude. Pilot study was conducted among ten nos. of population prior actual study. Questionnaire was revised and finalized according to an input of the pilot study. The survey was conducted in month of January 2020 to February 2020. The survey was carried out in Pimpri-Chinchwad municipal corporation area of Pune city in Maharashtra, India. This questionnaire presented to the 100 numbers of peoples. The survey was conducted by visiting home of the surveyed population. Method used was to take face to face interview and seeks answers. Responses of individual questions were recorded on the questionnaire itself. Data gathered were sorted and analysed. Percentage of each question individually was determined. Moreover, to calculate the index of awareness, knowledge and attitude questions were divided into these three domains and presented in the table 2.

Table 1: Survey questionnaire

\begin{tabular}{|c|c|c|}
\hline Q. No. & List of questions & Option \\
\hline 1 & What are the symptoms of Dengue Fever? & a) Fever b) Skin Rash c) Bleeding from nose d) All of the above \\
\hline 2 & Do you know about transmission of Dengue? & $\begin{array}{l}\text { a) Mosquito bite b) Don't know } \\
\text { c) Sharing food with infected people }\end{array}$ \\
\hline 3 & Which are the Breeding sites of Mosquitoes? & a) Boxes, Cans, Pots, Water containers b) Ponds., River c) Forest \\
\hline 4 & What are the sources of information? & $\begin{array}{l}\text { a) Health personal b) Radio/TV c) Newspaper/Magazine d) Relatives } \\
\text { and Friends (Any one or more) }\end{array}$ \\
\hline 5 & Do you know about Biting time of mosquitoes? & a) Dawn b) Morning c) Daytime d) Any time e) Night \\
\hline 6 & Attitude of responders. When he understood that he/she is ill.? & a) See a Physician b) See a Traditional (Ayurvedic and Homeopathic) \\
\hline 7 & Who should be responsible for mosquito's control? & a) Government b) Yourself c) Both \\
\hline 8 & Whether the Dengue Fever recurrent (person may get it again)? & a) Yes b) No c)Don't Know \\
\hline 9 & What you do in first stage of Fever? & $\begin{array}{l}\text { a) Treat myself at Home b) Don't Know } \\
\text { c) Take Traditional medicine (Ayurvedic and Homeopathic) }\end{array}$ \\
\hline 10 & 10. A) Do you store water at Home? & a) Yes b) No \\
\hline 11 & What measures do you take to prevent Mosquitoes Bite.? & $\begin{array}{l}\text { a) Use mosquitoes Coil b) Use mosquitoes net c) Sprayed Insecticide } \\
\text { d) Use repellent. (Anyone or more) }\end{array}$ \\
\hline
\end{tabular}

Table 2: Questions groups for calculating each index

\begin{tabular}{lll}
\hline S. No. & Domains & Questions type \\
\hline 1 & Awareness about dengue & Q.3, Q.4, Q.7, Q.10 (a) and (b). \\
2 & Knowledge about dengue & $:$ Q.1, Q.2, Q.5 and Q.8 \\
3 & Attitude of responders & Q.6, Q.9 and Q.11 \\
\hline
\end{tabular}

\section{RESULTS AND DISCUSSION}

Following related survey were depicted to compare the finding of our study results. Knowledge, attitude and practice regarding dengue among people in Pakse Laos. This was a cross-sectional study conducted in nine villages of Pakse district in Champ sack Province during July to September 2006. The study population included people who had suffered From DF and/or were treated within less than two years. Almost all of the respondents were diagnosed from both symptoms and laboratory tests from the hospital. Persons who had not suffered from DF were excluded from the survey. The data were collected by a face-to-face interview with residents of households with a history of DF. The socio-demographic characteristics of these responders, among 230 eligible persons interviewed, 161 (70.9\%) were female and 69 (30.0\%) were male About $46.5 \%$ of them were in age group 31-45 $\mathrm{y}$, followed by $36.5 \%$ in the $15-30$ y age group. Among them 223 (97.0\%) were Buddhist, and only 7 (3.0\%) were Catholic. Among the Respondents, 81 (35.2\%) were in a preliminary school. Sixteen $(7.0 \%)$ had received a college [14]. Outlook of dengue in Malaysia Method-A cross-sectional study of dengue in peoples in gombae district, Malaysia Ariffin F., Ramlia S., Recognizing Life-threatening feature of dengue in peoples. It is cross sectional study involving old women and men as well as younger. Knowledge on lifethreatening symptoms, Knowledge of dengue and attitude of the peoples amongst dengue were collected using self-administered questions. The total responders were 866 , with $44.8 \%$ men and $55.2 \%$ women. The Knowledge score of dengue among responders was $800(30.6 \%)$ to average $(56.8 \%)$. It was observed that $600(27.5 \%)$ to average $46.3 \%$ responders have a positive attitude towards the dengue [15].

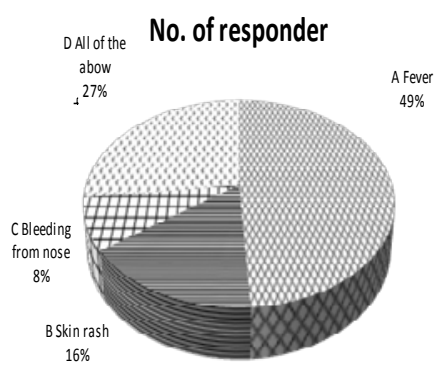

Fig. 1(Q.1): Symptoms of dengue fever

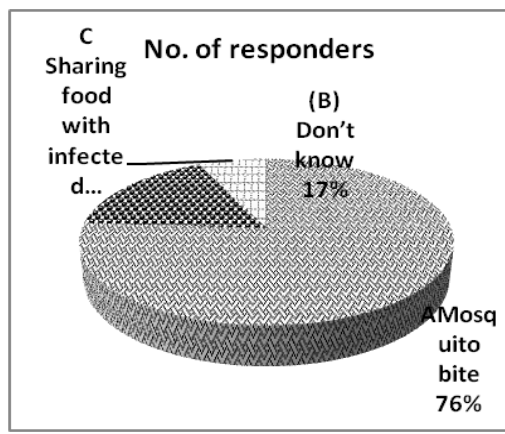

Fig. 2(Q.2): Transmission of dengue 


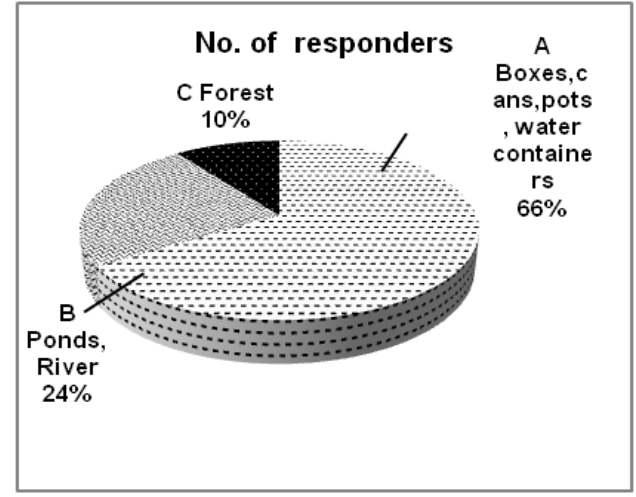

Fig. 3(Q.3): Breeding sites of mosquitoes

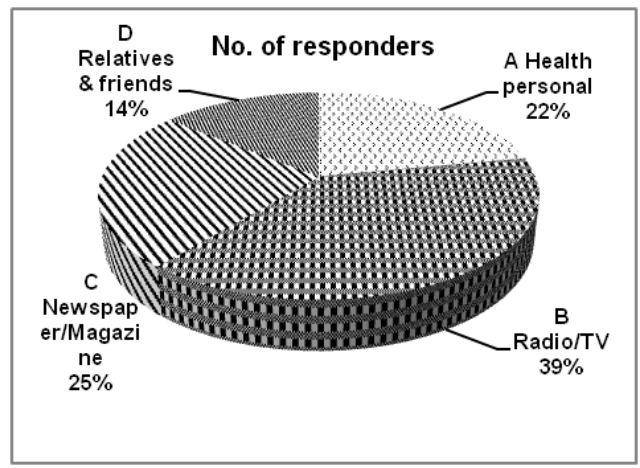

Fig. 4(Q.4): Sources of information

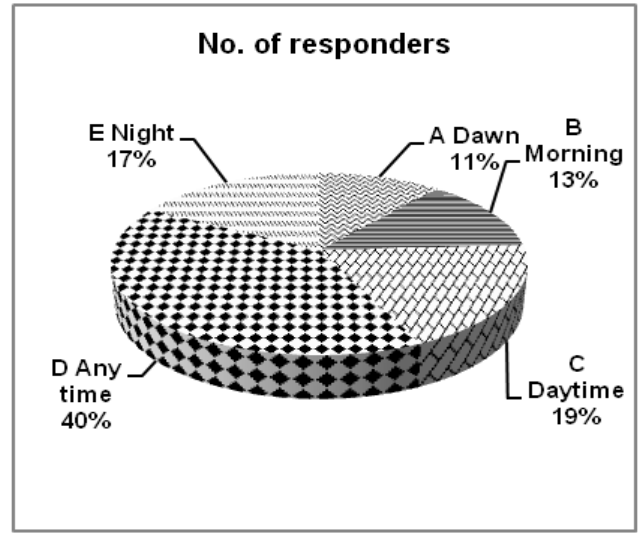

Fig. 5(Q.5): Biting time of mosquitoes

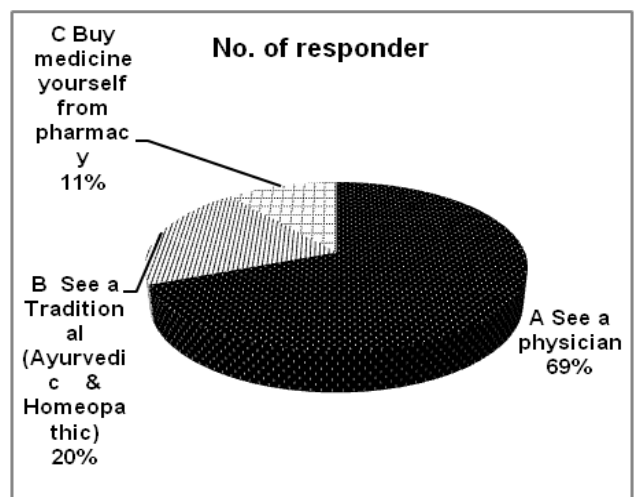

Fig. 6(Q.6): Attitude of responder when he understood that $\mathrm{He} / \mathrm{She}$ is ill

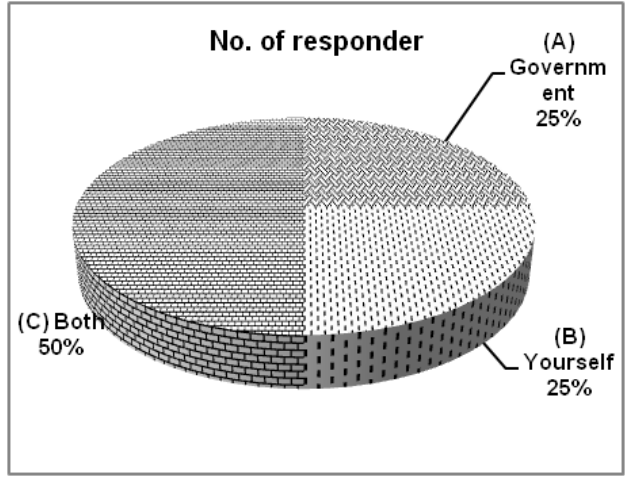

Fig. 7(Q.7): Responsibility for mosquito's control

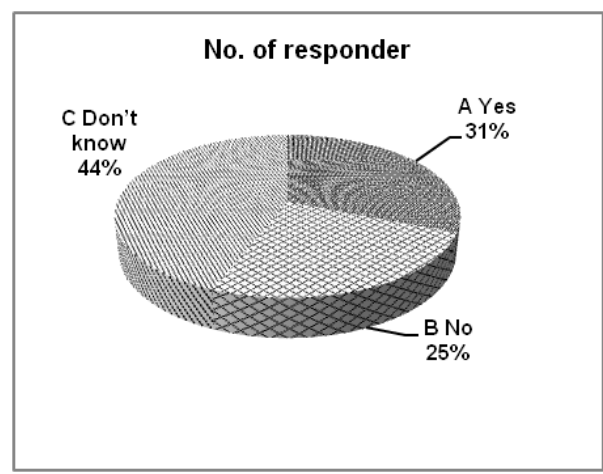

Fig. 8(Q.8): Recurrence possibility of dengue

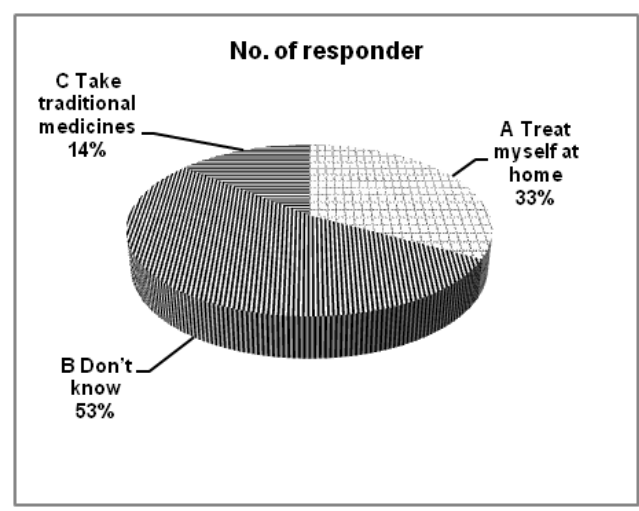

Fig. 9(Q.9): Attitude in the first stage of fevers

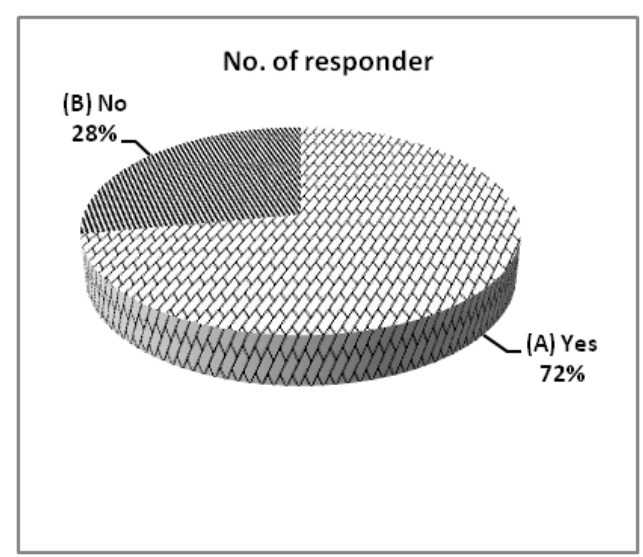

Fig. 10(Q.10A): Storage of water 


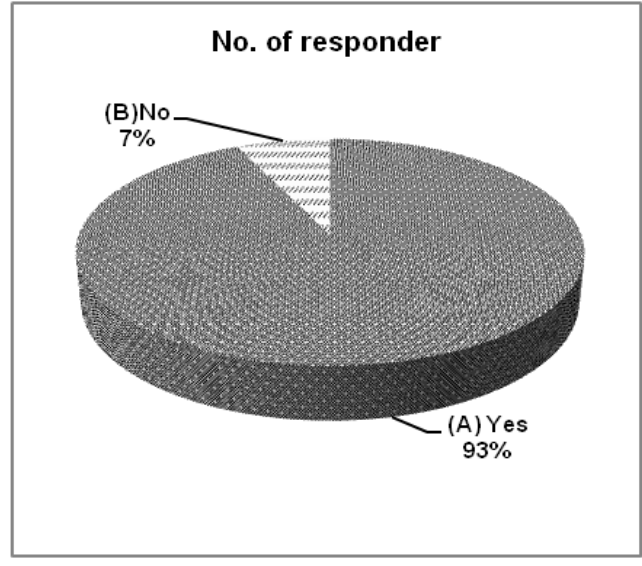

Fig. 11(Q.10B): Action for stored water

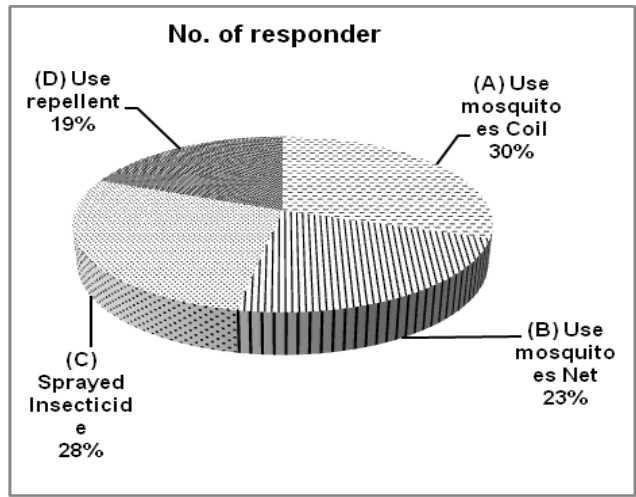

Fig. 12(Q.11): Prevention of mosquitoes bite

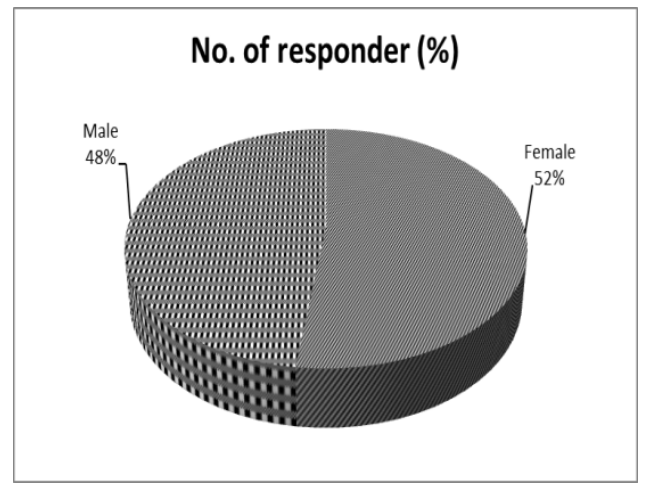

Fig. 13: Percentage according to male and female category

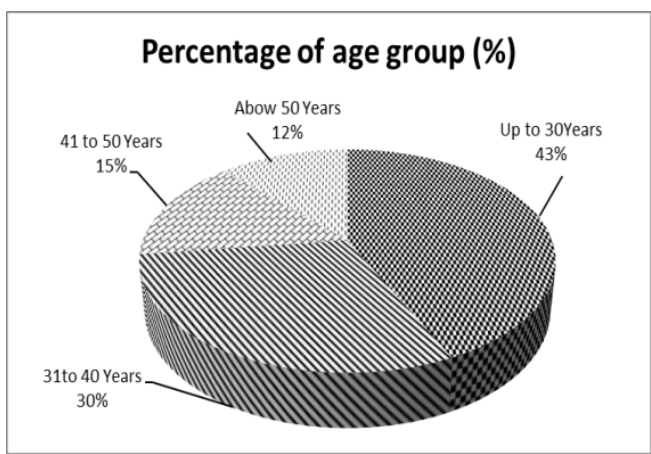

Fig. 14: Percentage of age group

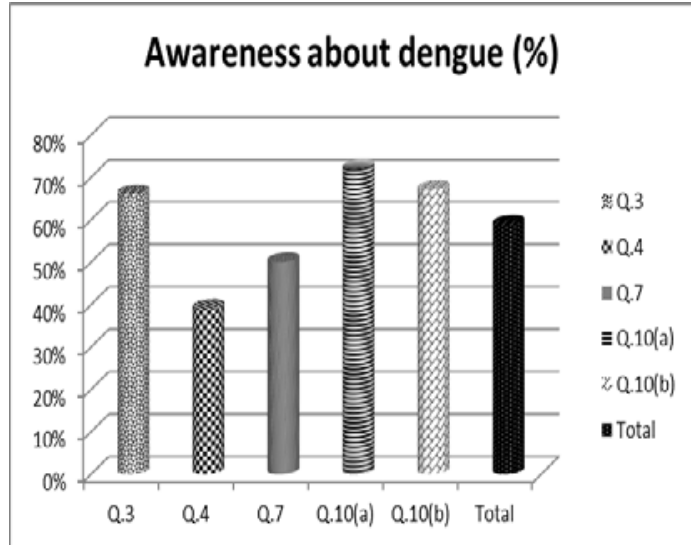

Fig. 15: Awareness about dengue

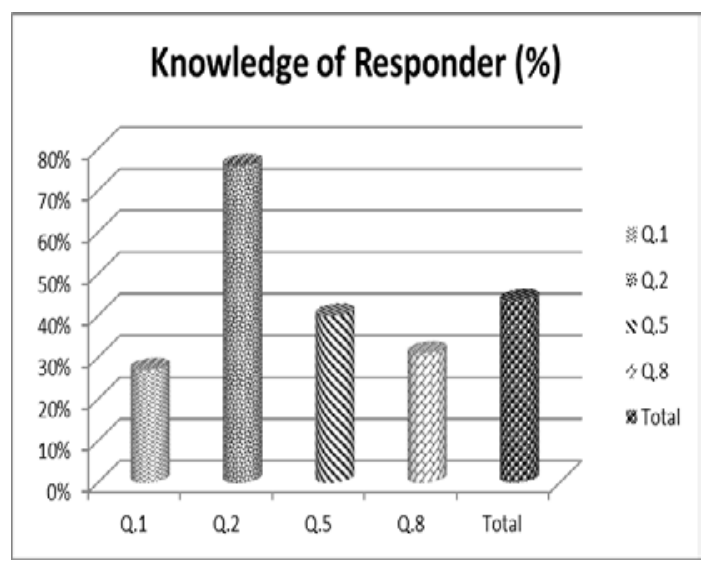

Fig. 16: Knowledge about dengue

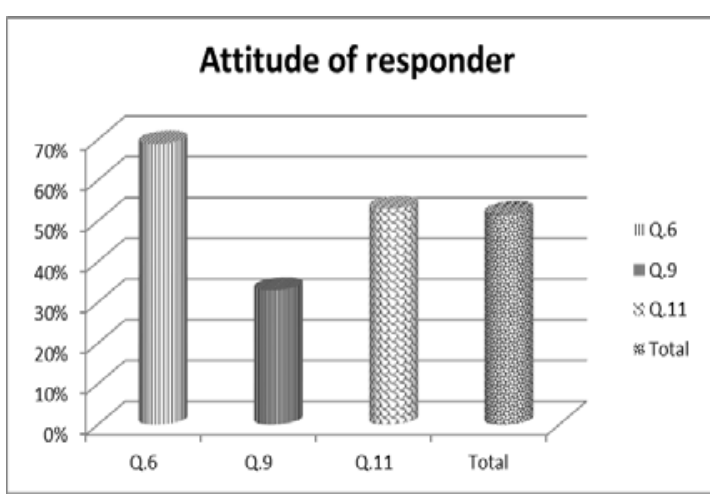

Fig. 17: Attitude of responders (\%)

As compare to previous studies; it was observed regarding response for breeding site of mosquito $66 \%$ peoples says Box, Cans, Pots, Water containers, $24 \%$ peoples says Ponds, River and $10 \%$ peoples says Forest. and the mosquitoes most breed from Boxes, cans, pots, and water containers. It means $66 \%$ surveyed population were aware risk of stored water can proliferate mosquito and lead to spread of Dengue. It was observed for sources of information of dengue: $22 \%$ peoples were become aware by health personnel, $39 \%$ was aware from Radio/TV, $25 \%$ peoples were aware from newspaper/magazine and $14 \%$ peoples were aware from relatives and friends. All three sources played an important role in creating awareness among society. It was found that $25 \%$ peoples say government are responsible for mosquitoes control, 25\% peoples says self's are responsible for that and $50 \%$ peoples says both are 
the responsible for mosquitoes control. These peoples should be made aware that its mutual responsibility. It was found that $72 \%$ peoples stored water at home and $28 \%$ of peoples not stored water at home but from these $67 \%$ peoples was frequently changed this stored water and it was the good attitude means $67 \%$ peoples were aware regarding the spreading of infection due to accumulated water. Regarding symptoms of dengue fever $49 \%$ people says fever, $16 \%$ people says skin rash, $8 \%$ people says bleeding from nose and $27 \%$ people says all of the above. Majority of surveyed population were aware about the distinguishing symptom of dengue that high fever. The majority was given to the fever, but the correct answer was all of the above. About transmission of dengue $76 \%$ peoples says mosquitoes are responsible, $17 \%$ peoples don't knew about this and $7 \%$ peoples says, by sharing the food with infected people dengue are transmitted. There were $11 \%$ peoples says biting time of mosquito is dawn, $13 \%$ says morning, $19 \%$ says daytime, $40 \%$ says any time and $17 \%$ peoples says at night mosquitoes bites. If the person was affected by dengue in past then possibility may get it again because dengue is the mosquito-borne disease and mosquitoes may bite to the person any time. Regarding this $31 \%$ peoples says person may get it again, $25 \%$ peoples says it does not get it again and $44 \%$ peoples says don't know about this. If person understood he/she infected by dengue, then $69 \%$ peoples see a physician, 20\% of peoples see a traditional (Ayurvedic and Homeopathic) and $11 \%$ buy medicine on their own from pharmacy. Significant surveyed population had the correct knowledge that they should see physician if they get symptoms of Dengue. In first stage of dengue $33 \%$ peoples treat our self's at home, 53\% peoples don't knew what to do and $14 \%$ peoples take traditional medicines [1518]. For the prevention of mosquitoes Bite $30 \%$ peoples used mosquito coil, $23 \%$ peoples used mosquito Net, $28 \%$ peoples spray insecticide and $19 \%$ peoples used repellent. It is found out that caution was taken by population. Index of awareness, knowledge and attitude was found to be $59 \%, 44 \%$ and $52 \%$, respectively. Results are represented in the fig. 1 to 18.

\section{ACKNOWLEDGEMENT}

Authors are grateful to Dr. Manohar J. Patil, Principal, Marathwada Mitra Mandal's College of Pharmacy, Pune for providing the facility to conduct the study.

\section{FUNDING}

Nil

\section{AUTHORS CONTRIBUTIONS}

All the authors have contributed equally.

\section{CONFLICT OF INTERESTS}

Declared none

\section{REFERENCES}

1. Poddock M. World Health Organization. Global Alert and Response. Impact of Dengue; 2013.

2. World Health Organization. Dengue and dengue hemorrhagic fever. Fact Sheet No. 117. WHO, Geneva; 2002.

3. Behrens R, Carroll B. Dengue infections and travel. British travel health association, Newsletter travel wise; 1991. p. 4-5.

4. Gubler DJ. Dengue and dengue hemorrhagic fever. Clin Microbial Rev 1998;11:480-96.

5. Brady OJ, Gething PW, Bhatt S. Refining the global spatial limits of dengue virus transmission by evidence-based consensus. Nature 2013;496:504-7.

6. Guglani L, Kabra SK. T cell immunopathogenesis of dengue virus infection. Dengue Bull 2005;29:58-68.

7. Massad E, Rocklov J, Wilder Smith A. Dengue infections in nonimmune travellers to Thailand. Epidemiol Infect 2013;141:412-7.

8. Laos National Textbook for Treatment Guideline; Ministry of Health, Laos PDR; 2001. p. 45-78.

9. National Institute of Public Health. Health status of the people in Laos PDR. 37, Vientiane, Laos; 2001.

10. Mahlaath S. Training manual planning and investment department, Champasack Province, Laos; 2005. p. 4-6.

11. Schmidt Chanasit J, Haditsch M, Schönenberg I. Dengue virus infection in a traveller returning from croatia to germany. Euro Surveil 2010;15:19677.

12. Krishnamoorthy Y, Chander D. Household survey on public awareness and attitude towards dengue infection in rural Tamil Nadu, south India. J Education Health Promotion 2018;7:171.

13. Sagunadevi G, Dharmaraj A. An awareness program on dengue fever among adults residing in and urban slum area coimbtore. Int J Res Med Sci 2017;12:5242-6.

14. Thomas SJ, Stickman D. Oxford journals medicine and health. J Inf Disease 1998;198:948-61.

15. P Gautret P, Cramer JP, Field V. Infectious diseases among travelers and migrants in Europe. Eurosurveillance 2012;17:16-26.

16. Nalongsack S, Yoshida Y, Morita S, Sosouphanh K, Sakamoto J, Knowledge Attitude, et al. Regarding dengue fever in Pakse, Laos, Nagoya J. Med Sci 2009;71:29-37.

17. Rudnick A, Tan E, Lukas JK, Omar M. Mosquito borne fever in Malaysia, Malaysian. Pathole J 1965;1:1269-72.

18. Sinha K. World Health TNN; 2015. 